\title{
MODEL BASED PREDICTIVE CONTROL USING NEURAL NETWORK FOR BIOREACTOR PROCESS CONTROL
}

\author{
Laurentiu FRANGU, Sergiu CARAMAN, Emil CEANGA,
}

Department of Automatic Control and Electronics,

"Dunarea de Jos" University, Domneasca 111, 6200 Galati, Romania

phone/fax: +40 36460 182, email: Sergiu.Caraman@ugal.ro

\begin{abstract}
This paper deals with a neural network based GPC structure for a bioprocess control. Comparing to IMC structure, this method offers two advantages: the neural inverting operation of the process model is eliminated and there are various possibilities to adjust the control law properties. The GPC method is applied to a biomass production process and to an enzymatic production process (lipase producing). In both cases many simulation results are presented which illustrate the validity of the method.
\end{abstract}

Keywords: predictive control, neural network, training, bioreactor, bioprocess.

\section{INTRODUCTION}

The complexity of the bioprocesses makes their control problem very difficult. The usual modeling procedures, based on kinetic enzymatic schemes, lead to non-linear state models, with a large number of parameters. Moreover, the state variables that characterize the proper biosynthesis process are not accessible to the direct measurement. In the basic configuration, the bioreactor has control loops only for the physical and chemical parameters of the culture environment (temperature, stirring, aeration, $\mathrm{pH}$, etc.). This fact has determined the development of some specific techniques to estimate the parameters and for the state observers synthesis (Bastin et al., 1990). Since the bioprocesses have frequently a variant character, the techniques mentioned above are used in the context of the linearizing adaptive control methods (Bastin et al., 1990)
The capacity of the neural networks (NN) for the complex input/output models identification has stimulated their usage in the bioreactors control structures. One of the basic approaches, concerning the neural techniques in the bioprocesses control, is Internal Model Control (IMC) Framework (Garcia et al., 1982). Neural network model - based IMC structure involves the usage of two neural networks, trained for the determination of both direct and inverse models. Assuming the direct model is ideal, the comparison of its output to the one of the process allows the disturbance reconstruction. The inverse model is the controller, performing the functions of reference tracking and disturbance rejection.

A large class of neural network model - based control structures could be framed in the domain of ModelBased Predictive Control (MBPC) (De Keyser, 1991; Camacho et al., 1999). The 
present paper deals with a biosynthesis process control, using the Generalized Predictive Control (GPC) structure (Clarke et al., 1989), which is part of MBPC approach. Comparing to the IMC approach, the main arguments that justify the usage of GPC structure, in the framework of the biosynthesis processes neural control, are given as follows:

1. The neural inverting operation of the process model, needed for the controller implementation, is eliminated. As it is well known, the inverse neural model training is always more difficult than the one of the direct model (Bhat et al., 1990). In the case of the biosynthesis processes, where the model complexity is high, the training of the inverse model (non-causative) could involve difficulties and risks, especially in adaptive context. This is the reason why in the IMC, using neural networks approach, hybrid models are proposed. They fructify some $a$ priori information regarding the model. Thus the neural identification operates with a "gray box" instead of a "black box" (Aoyama et al., 1995). This solution reduces in a certain range the difficulties of the inverse model training, without their elimination, whereas GPC approach eliminates the inverse model.

2. GPC offers various possibilities for the control law adjustment by means of the following parameters: the prediction horizon, the control horizon, the weights of the error and the command. The control feasibility requirements make these instruments very useful for the control law adjustment.

The paper analyses the properties of the neural network based GPC structure for a bioprocess control in two variants:

1. using continuous command

2. using discrete command.

The paper also illustrates some results regarding the usage of this structure to the lipase biosynthesis process optimization.

The structure of the paper is as it follows: the next section contains an introduction in GPC structure. The third section presents some basic results referring to the neural network modelbased control in the GPC structure, for a biotechnological process, such as: the reference tracking, the disturbance rejection, the modification of the control law properties by adjusting the parameters which appear in the performance criterion. The fourth section deals with the same problems from the previous section, using the GPC structure with discrete command. Section 5 describes the performances of GPC approach in a case study concerning the optimization of the lipase biosynthesis process. A summary and conclusions are given in the final section.

\section{GENERALIZED PREDICTIVE CONTROL}

From the variety of MBPC strategies, the most spread is GPC algorithm, illustrated in Figure 1.

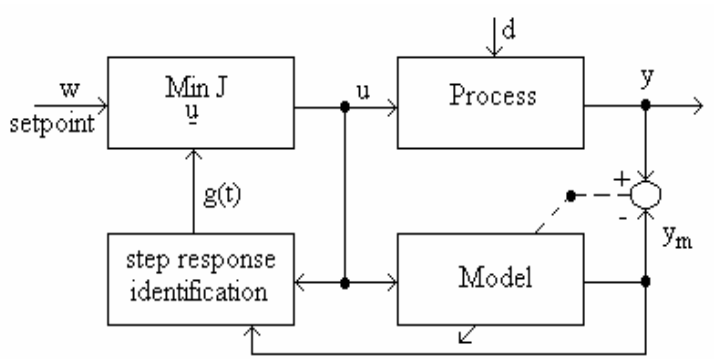

Fig. 1. GPC structure

Let us consider $t$ the discrete current time in GPC procedure. The command variation at the current step $\Delta u(t)$ is deduced from the condition of the criterion minimization:

$$
\begin{array}{r}
J=\sum_{k=N_{1}}^{N_{2}} \delta^{2}(k) \cdot[w(t+k)-y(t+k / t)]^{2}+ \\
+\sum_{k=0}^{N_{u}-1} \lambda^{2}(k) \cdot[\Delta u(t+k / t)]^{2}
\end{array}
$$

where $y(t+k / t)$ is $k$-step ahead prediction of the system output on data up to time $t ; N_{1}$ and $N_{2}$ the minimum and maximum prediction horizons; $N_{u}$ - the control horizon; $w(t+k)$ - the future setpoint; $\delta(k)$ and $\lambda(k)$ - the weighting coefficients of the errors and of the commands, respectively. The internal model, assumed to be perfect, is used to determine the predictions $y(t+k / t)$. At every step of the GPC procedure, the unitary step response of the internal model in zero initial conditions is determined. For this, it is necessary to admit that the model could be linearized around the current working point. The step response identification involves the following operations: 
- the previous command is kept constant and the free response is determined: $y_{\text {free }}(t+k / t)$, $k=1, \ldots, N_{2}$;

- the step response of the model is determined: $y(t+k / t), k=1, \ldots, N_{2}$.

Since

$$
y(t+k / t)=y_{\text {free }}(t+k / t)+y_{\text {forced }}(t+k / t)
$$

with

$$
\begin{gathered}
y_{\text {forced }}(t+k / t)=\sum_{i=1}^{k} g_{i} \cdot \Delta u(t+k-i / t), \\
k=1, \ldots, N_{2}
\end{gathered}
$$

results

$$
\sum_{i=1}^{k} g_{i} \cdot \Delta u(t+k-i / t)=y(t+k / t)-y_{\text {free }}(t+k / t)
$$

where $g_{i}$ represent the step response coefficients in zero initial conditions.

Replacing consecutively in relation (4) $k$ with $\left(1,2, \ldots, N_{2}\right)$ the values $g_{1}, g_{2}, \ldots, g_{N 2}$ are obtained. Within the prediction horizon $\left[N_{1}, N_{2}\right]$, the linearized model can be written as it follows:

$$
Y=G U+Y_{\text {free }}
$$

where

$$
\begin{aligned}
& Y=\left[y\left(t+N_{1} / t\right), y\left(t+N_{1}+1 / t\right), \ldots, y\left(t+N_{2} / t\right)\right]^{T} \\
& U=\left[\Delta u(t / t), \Delta u(t+1 / t), \ldots, \Delta u\left(t+N_{u}-1 / t\right)\right]^{T} \\
& Y_{\text {free }}=\left[y_{\text {free }}\left(t+N_{1} / t\right), y_{\text {free }}\left(t+N_{1}+1 / t\right), \ldots\right. \\
& \left.\ldots, y_{\text {free }}\left(t+N_{2} / t\right)\right]^{T} \\
& G=\left[\begin{array}{cccc}
g_{N_{1}} & g_{N_{1}-1} & \ldots & 0 \\
g_{N_{1}+1} & g_{N_{1}} & \ldots & 0 \\
\vdots & \vdots & \vdots & \vdots \\
g_{N_{2}} & g_{N_{2}-1} & \ldots & g_{N_{2}-N_{u}-1}
\end{array}\right]
\end{aligned}
$$

Using the model (5) in equation (1), a quadratic form for the performance criterion, with respect to $U$, is obtained:

$$
\begin{aligned}
J= & {\left[\Delta \cdot W-\Delta \cdot\left(G U+Y_{\text {free }}\right)\right]^{T} \cdot[\Delta \cdot W-} \\
& \left.-\Delta \cdot\left(G U+Y_{\text {free }}\right)\right]+(\Lambda U)^{T}(\Lambda U)
\end{aligned}
$$

where

$$
W=\left[w\left(t+N_{1} / t\right), w\left(t+N_{1}+1 / t\right), \ldots, w\left(t+N_{2} / t\right)\right]^{T}
$$

$$
\Delta=\operatorname{diag}\left[\delta(1), \ldots, \delta\left(N_{2}-N_{1}+1\right)\right]
$$

$$
\Lambda=\operatorname{diag}\left[\lambda(1), \ldots, \lambda\left(N_{u}\right)\right]
$$

From the condition of the criterion minimization, the following control law is obtained:

$$
U=\left[G^{T} \cdot \Delta^{2} \cdot G+\Lambda^{2} \cdot I\right)^{-1} \cdot \Delta \cdot G\left(W-Y_{\text {free }}\right)
$$

Only the first component of the vector $U$, $\Delta u(t / t) \equiv \Delta u(t)$, is applied. Shifting to the future the prediction and control horizons, the next step of the algorithm is prepared. Then, the algorithm operations are repeated.

\section{STABILIZATION AND TRACKING REGIME OF A BIOREACTOR CONTROLLED BY GPC ALGORITHM}

\subsection{The neural model of the bioprocess}

For testing the GPC algorithm, the bioprocess presented in (Aoyama et al., 1995) has been considered. In the paper mentioned above the performances of the IMC procedure was determined. This alternative has permitted to draw some comparative conclusions regarding the two control procedures. The process takes place in a continuous stirred bioreactor and it is described by the following equations:

$$
\begin{aligned}
& \dot{X}=(\mu-D) \cdot X \\
& \dot{S}=D \cdot\left(S_{f}-S\right)-\frac{1}{Y_{X / S}} \cdot \mu \cdot X \\
& \dot{P}=-D \cdot P+(\alpha \cdot \mu+\beta) \cdot X
\end{aligned}
$$

with

$$
\mu=\frac{\mu_{\max } \cdot\left(1-P / P_{m}\right) \cdot S}{K_{m}+S+S^{2} / K_{i}}
$$


where

$X=$ cell mass concentration $(5.9956 \mathrm{~g} / \mathrm{l}$ at the steady-state);

$P=$ product concentration $(19.1267 \mathrm{~g} / \mathrm{l}$ at the steady-state);

$S=$ substrate concentration in the culture $(5.0109 \mathrm{~g} / \mathrm{l}$ at the steady-state);

$S_{f}=$ feed substrate concentration in the culture $(20.0 \mathrm{~g} / \mathrm{l}$ at the steady-state);

$D=$ dilution rate $(0.202 \mathrm{l} / \mathrm{h}$ at the steady-state);

$\mu=$ specific growth rate $[1 / \mathrm{h}]$ given by (18), which includes the inhibition due to the substrate and the reaction product;

$Y_{X / S}=$ cell mass yield $(0.4 \mathrm{~g} / \mathrm{g})$;

$\alpha=$ kinetic parameter $(2.2 \mathrm{~g} / \mathrm{g})$;

$\beta=$ kinetic parameter $(0.2 \mathrm{l} / \mathrm{h})$;

$\mu_{\max }=$ maximum specific growth rate $(0.48 \mathrm{l} / \mathrm{h})$;

$P_{m}=$ product saturation constant $(50 \mathrm{~g} / \mathrm{l})$;

$K_{m}=$ substrate saturation constant $(1.2 \mathrm{~g} / \mathrm{l})$;

$K_{i}=$ substrate inhibition constant $(22 \mathrm{~g} / \mathrm{l})$.

The non-linear behavior of the process clearly results from its static characteristic presented in Figure 2.

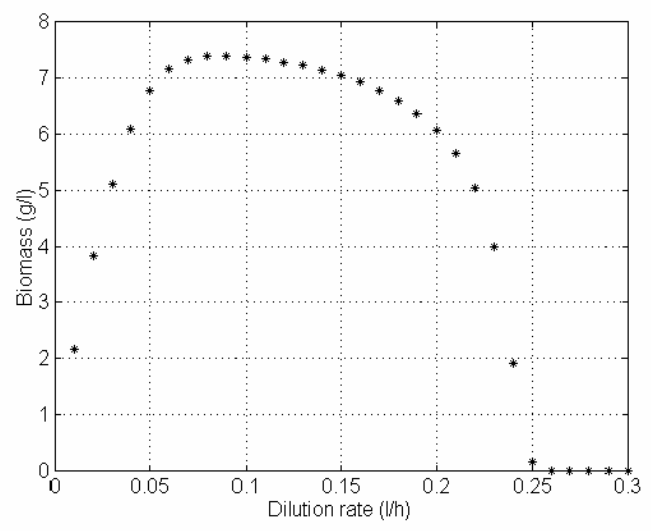

Fig. 2. The static characteristic of the process

A structure with three layers has been proposed as internal neural model: four input neurons, seven sigmoidal neurons in the hidden layer and one linear neuron as output layer (Figure 3). The neural network output is the biomass increment. For the neural network training both the transient and the quasi - steady state data were used. The transient data have been obtained by integrating the differential equations (15) - (17), considering the randomly varying dilution rate within range $\pm 50 \%$ around the steady-state value (Figure 4). The quasi - steady state data has been obtained by integrating equations (15) - (17) considering different values of the dilution rate $D$ (Figure 5).

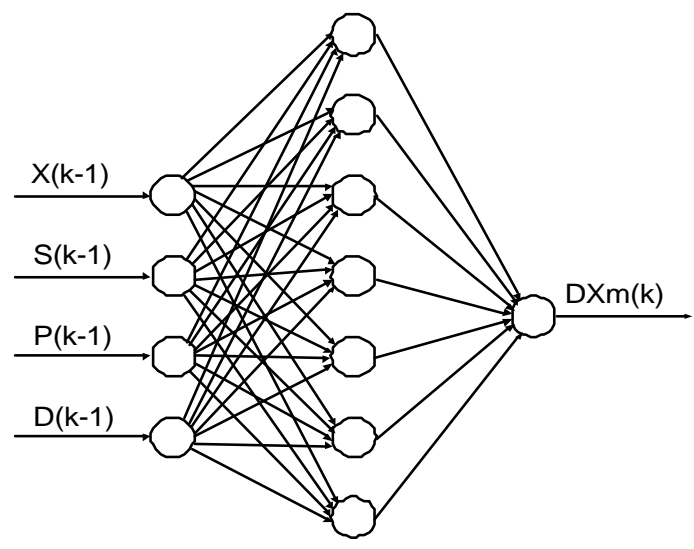

Fig. 3: The neural network structure
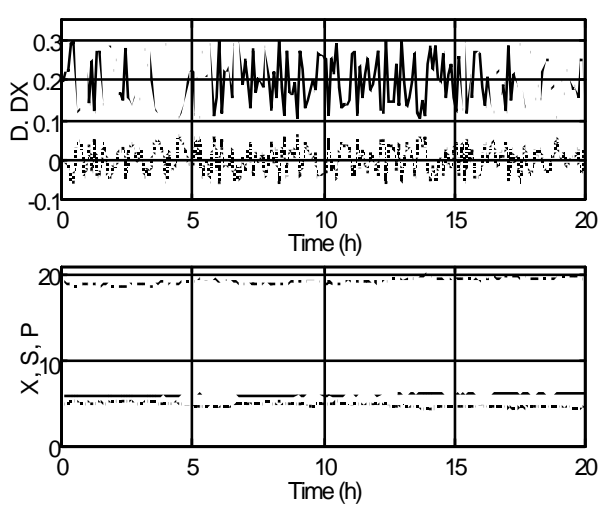

Fig. 4. Transient data used for neural network training
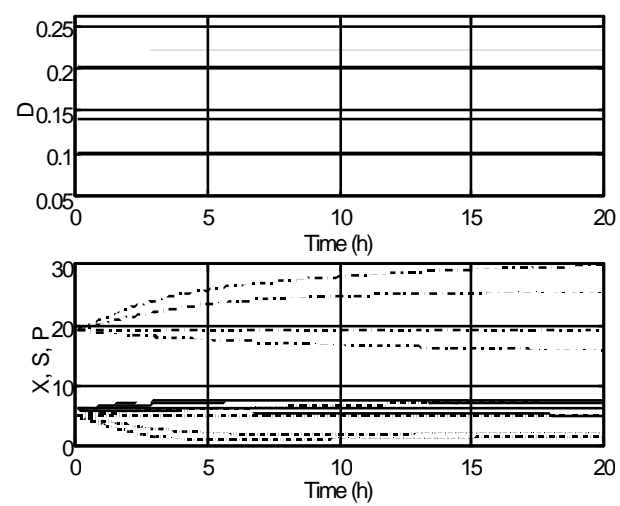

Fig. 5. Steady - state data used for neural network training

The validation of the neural network training has been made with test data, which were not used during the training (Figure 6). We can see that the network leads to a very good approximation, the difference between the process output and the model being almost imperceptible.

The testing of the process described by the equations (15)-(17) and of the neural model with 
a multi-step sequence shows that the approximation is very good for the biomass values within the range [3.5-8] g/l (Figure 7). For values of the biomass that exceed the domain of the training set (e.g. less than $3.5 \mathrm{~g} / \mathrm{l}$ ), the error of the neural network model output becomes larger (Figure 7).

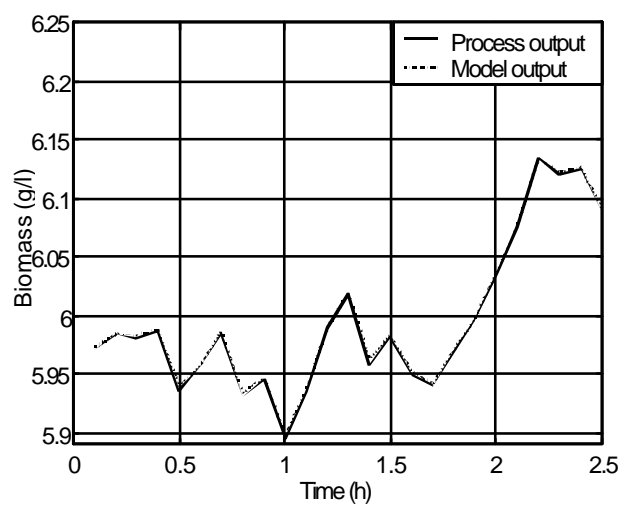

Fig. 6. The validation of the neural network

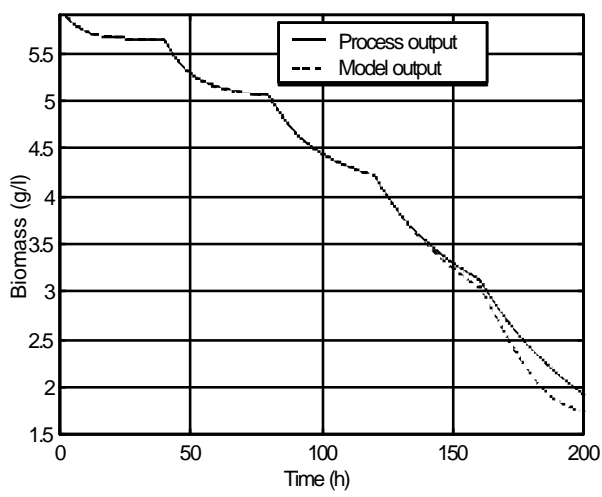

Fig. 7. The process and model response when the input is a multistep sequence

\subsection{The performances of the closed-loop system}

The control objective is to maximize the output rate of biomass per unit volume of culture. To reach the optimum, the controller has to keep the process in a functioning point $(D, X)$, determined from the static characteristic (Figure 2). Consequently, the performances of the closed-loop system were studied through variations around the chosen steady functioning point.

A step setpoint from 6 to 5 1/h has been applied to the system presented in Figure 1. For the choice of the step value, the following issues were taking into account:

- the working point has to lie in the range where the neural model has been trained;
- the command $D$ should not exceed the admissible interval.

Further on, the following parameters of the predictive controller were successively varied:

1. The prediction horizon $\left(N_{2}\right)$ (the other parameters were kept constant to the values: $N_{u}=1, \lambda=10$ and $\delta=1$. In Figure 8 it can be seen that the process output reaches the setpoint more slowly and the command has smaller variations as the prediction horizon increases.

2. The control horizon $\left(N_{u}\right)$ (the other parameters were kept constant to the values: $N_{2}=8, \lambda=10$ and $\delta=1$. Figure 9 shows that the increasing of the control horizon value leads to the increasing of the stabilization speed of the process output to the setpoint.

3. The penalty factor of the command variations $(\lambda$-parameter, $\lambda(k)=\lambda)$; the other parameters were kept constant to the values: $N_{2}=4, N_{u}=1$ and $\delta=1$ - Figure 10.

4. The penalty factor of the future errors, which interfere in the criterion minimization ( $\delta$-parameter, $\delta(k)=\delta$ ); the other parameters were kept constant to the values: $N_{2}=4$, $N_{u}=1$ and $\lambda=10$ - Figure 11.

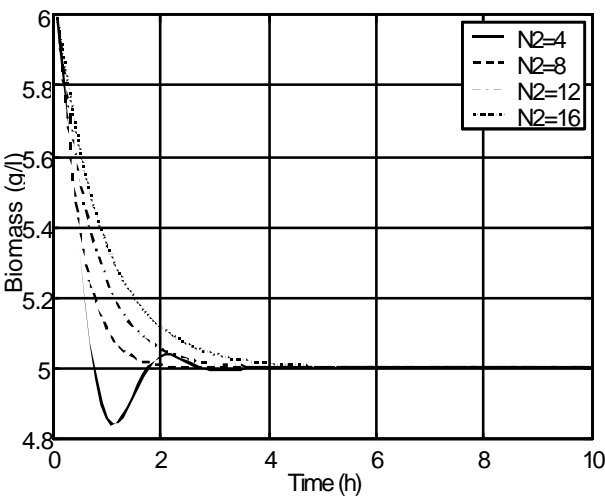

(a)

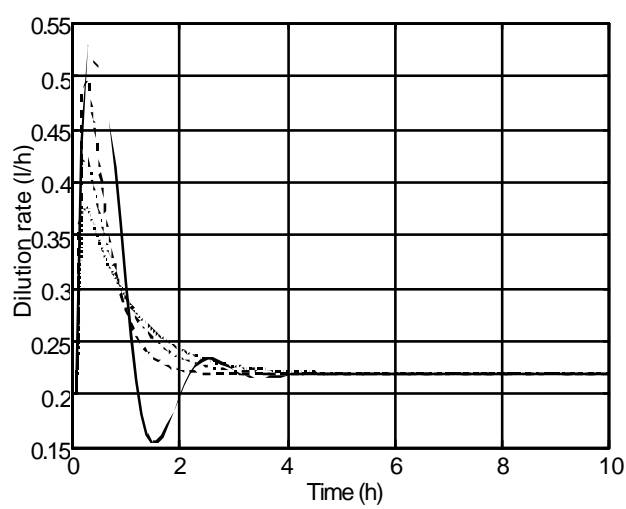

Fig. 8. The influence of $N_{2}$-parameter on the process ( $\mathrm{a}$-output and $\mathrm{b}$ - command) 


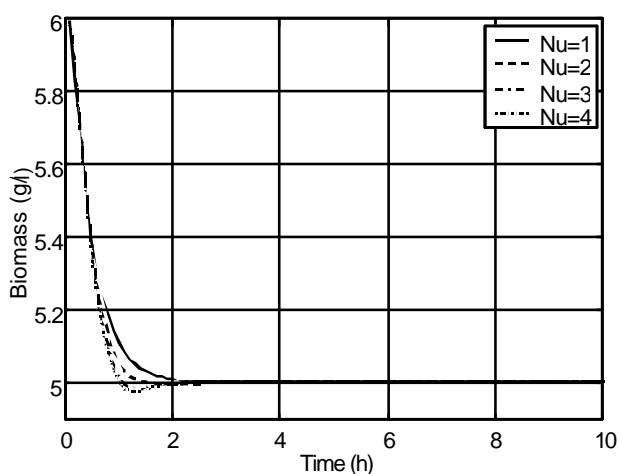

(a)

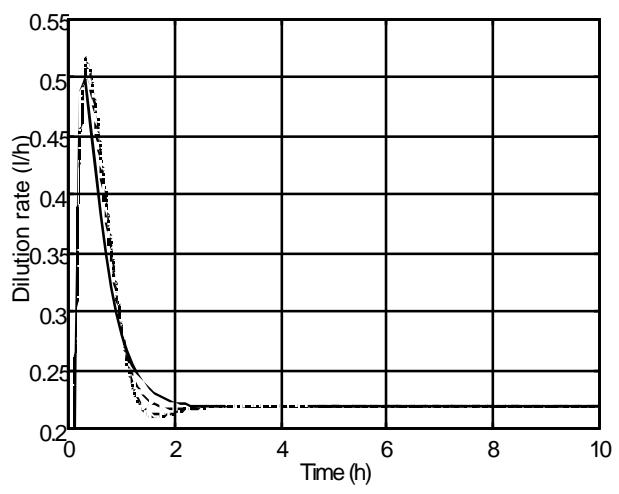

(b)

Fig. 9. The influence of $N_{u}$-parameter on the process ( $\mathrm{a}$ - output and $\mathrm{b}$ - command)

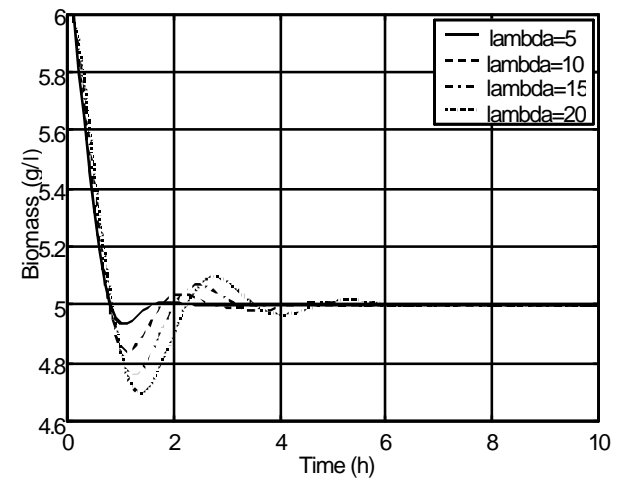

(a)

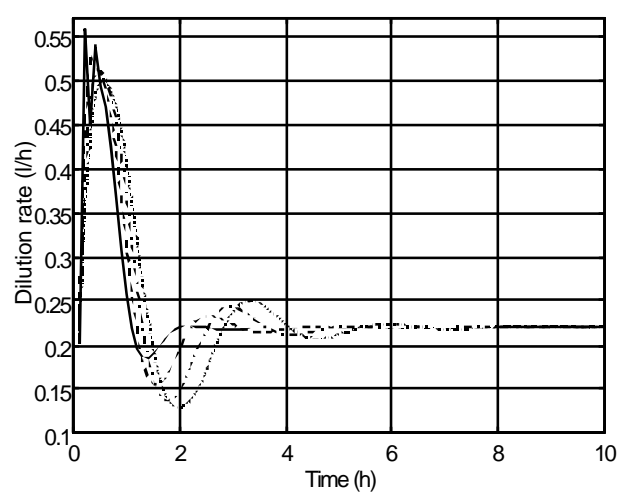

(b)

Fig. 10. The influence of $\lambda$-parameter on the process ( $\mathrm{a}$ - output and $\mathrm{b}$ - command)

Figures 10 and 11 show the influence of $\lambda$ and $\delta$ - parameters on the process output: as $\lambda$ increases or $\delta$ decreases, the overshot and the transient time become larger.

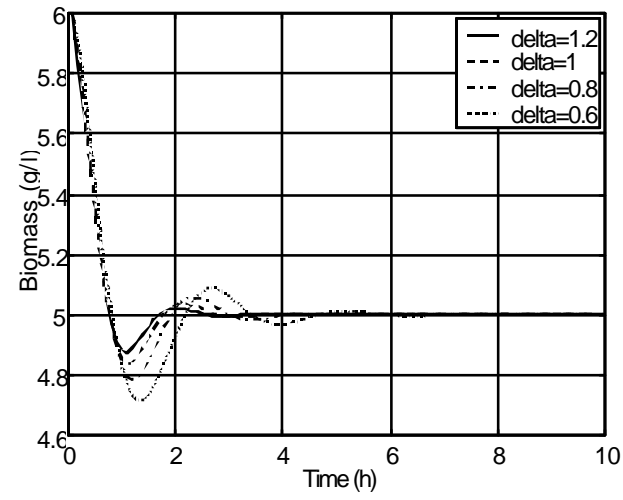

(a)

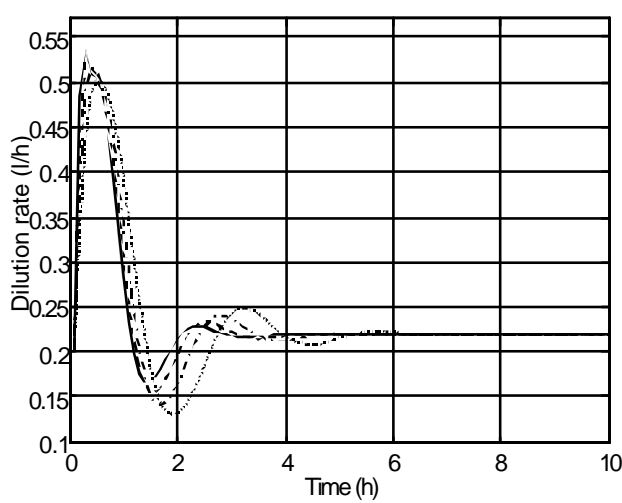

(b)

Fig. 11. The influence of $\delta$-parameter on the process ( $\mathrm{a}$ - output and $\mathrm{b}$ - command)

\subsection{Tracking performances of the closed-loop system}

The tracking properties of the system presented in Figure 1 were tested applying a quasi-periodic signal as setpoint. Taking into account the long duration of the considered bioprocess, the test signal frequency was chosen small enough so that the predictive algorithm could track the setpoint variations (Figure 12).

The system output magnitude varies within the range [2-8] g/l. These values are outside the domain where the neural model has a good behavior. The range [2-8] g/l was chosen to represent both zones: a good training zone and a poor training one. The increasing of the biomass concentration over $8 \mathrm{~g} / \mathrm{l}$ leads to unfeasible values for the command (less than 0 ). When the setpoint values are within the range [2-3] g/l, significant errors appear between the setpoint and the process output. This is due to the fact that within this range, the neural model does not approximate accurately enough the process output, as indicated in Figure 7. 


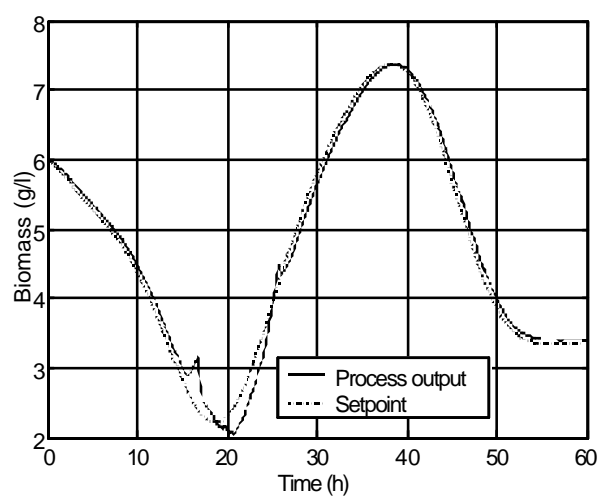

(a)

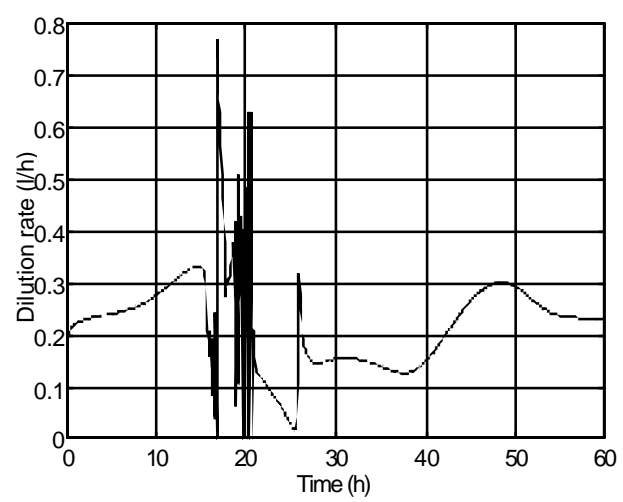

(b)

Fig. 12. The tracking behavior of the system (a -output and b-command)

\subsection{Disturbances rejection by the closed-loop system}

Two kind of disturbances were considered, affecting the feed substrate concentration $\left(S_{f}\right)$ and the specific growth rate $(\mu)$ :

1. A pseudo-random disturbance has been added to $S_{f}$, ranging within $\pm 25 \%$ interval (Figure 13).

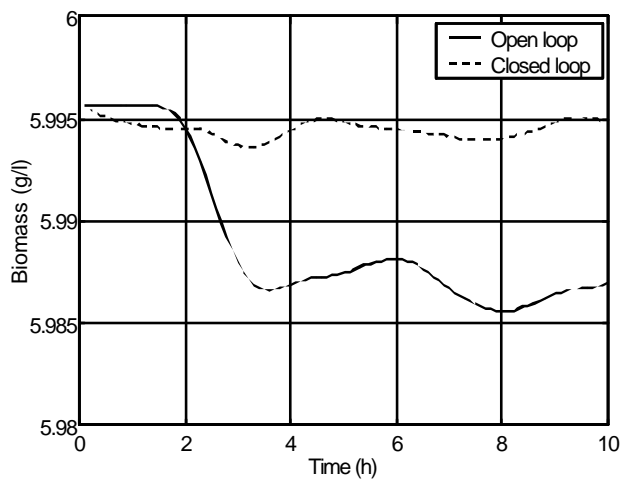

Fig. 13. Open-loop and closed-loop system behavior when $S_{f}$ is disturbed

2. After 2.5 hours, the value of $\mu$-parameter was increased with 50\% (Figure 14).

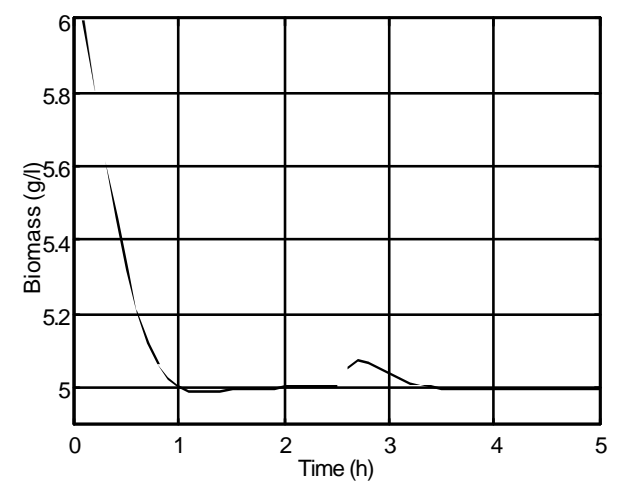

Fig. 14: The system behavior when $\mu$-parameter was modified

In both cases the control system rejected very well the disturbances.

\section{GPC STRUCTURE WITH DISCRETE COMMAND}

In many cases, the actuating equipment of the commands of a bioreactor can not track accurately the command $u(t)$ of the controller. Moreover, this equipment is often conceived to modify in steps the dilution rate so that the variable $D(t)$ can take only a reduced number of discrete values. In this case, the command variation $\Delta u(t)$ is found out from the criterion (1) minimization, searching through all the possible combinations of the discrete commands, on the control horizon - $N_{u}$. Obviously, for large values of the $N_{u}$ parameter, the computing time necessary to determine the optimal command could become prohibitive. For the bioprocess described in the previous section, the dilution rate was considered to take five possible values: $\{0 ; 0.05 ; 0.1 ; 0.15 ; 0.2\}$. For $N_{u}=1$, in Figures 15, 16 and 17 the closed loop performances of the system, when the prediction horizon $\left(N_{2}\right)$ takes the respective values 8,12 and 16, are illustrated. Figure 17 shows that the performances are satisfactory, even when using a reduced number of command commutations.

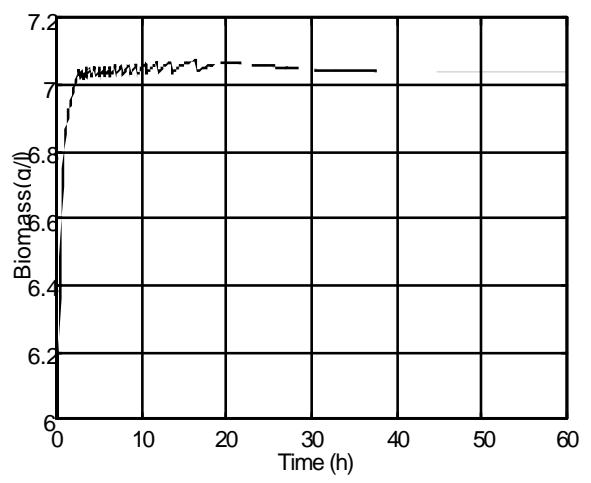




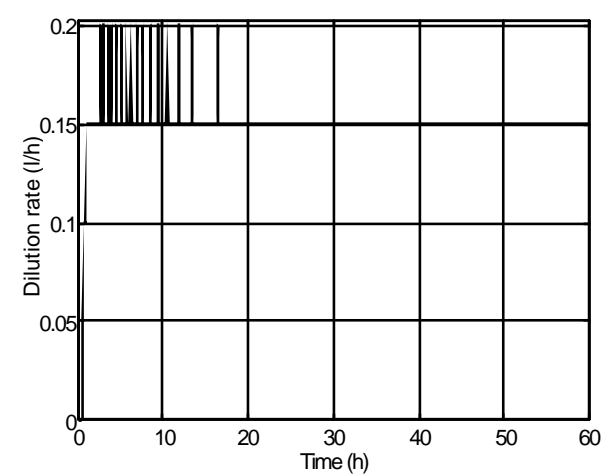

(b)

Fig. 15: The process behavior for $N_{2}=8$ (a output, $\mathrm{b}$ - discrete command)

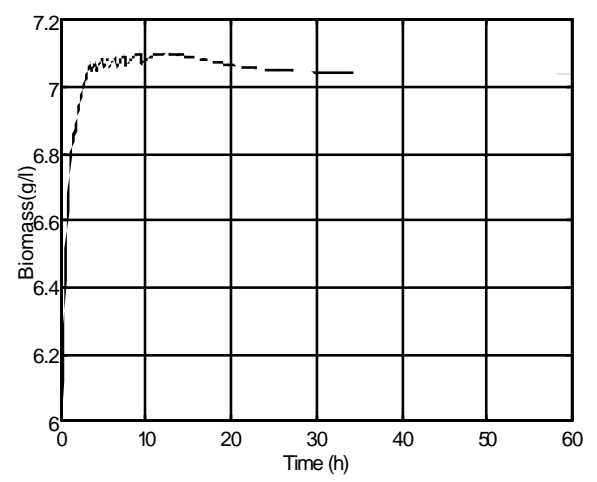

(a)

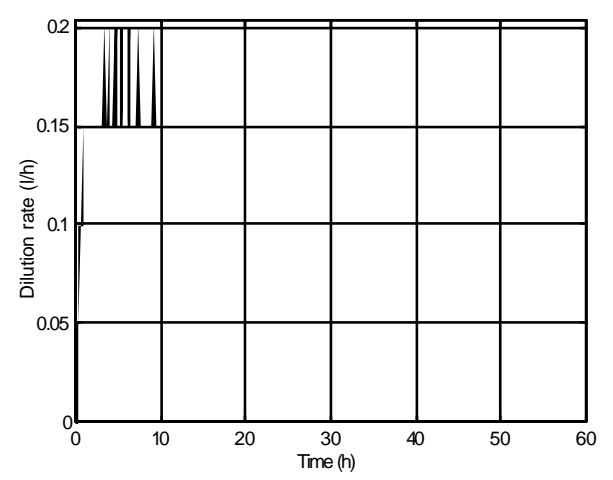

(b)

Fig. 16: The process behavior for $N_{2}=12$ (a output, b - discrete command)

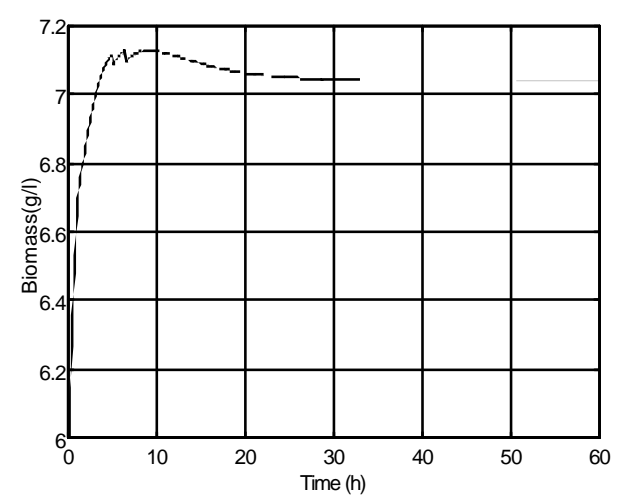

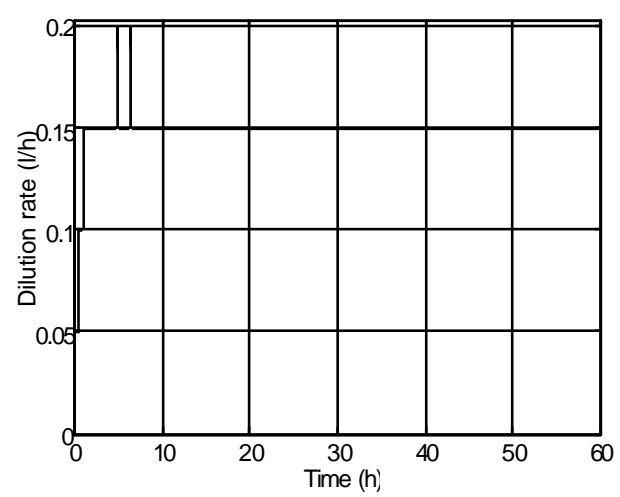

(b)

Fig. 17: The process behavior for $N_{2}=16$ (a output, b - discrete command)

\section{GPC OF LIPASE BIOSYNTHESIS PROCESS}

The lipase biosynthesis process is a very complex one, due to the simultaneous existence of more culture phases: liquid phase, organic phase, cellular phase and gaseous phase. The model describing this behavior is highly nonlinear and consists in the following differential equations (Selisteanu, 1999):

$$
\begin{aligned}
& \dot{S}_{1}=-\eta \cdot X+D \\
& \dot{S}_{2}=\eta-\mu \cdot\left(Y+S_{2}\right) \\
& \dot{X}=\mu \cdot X \\
& \dot{L}_{i n}=v_{p}-v_{e x}-\mu \cdot L_{i n} \\
& \dot{L}_{e x}=v_{e x} \cdot X
\end{aligned}
$$

where

$X=$ biomass concentration $[\mathrm{g} / \mathrm{l}]$;

$S_{1}, S_{2}=$ extracellular and intracellular substrate concentration $[\mathrm{g} / \mathrm{l}]$;

$L_{e x}, L_{i n}=$ extracellular and intracellular lipase concentration $[\mathrm{g} / \mathrm{l}]$;

$\eta=$ specific absorption rate of the substrate $\left[\mathrm{h}^{-1}\right]$; $\mu=$ specific growth rate of the biomass $\left[\mathrm{h}^{-1}\right]$;

$v_{p}=$ internal lipase production rate $\left[\mathrm{h}^{-1}\right]$;

$v_{e x}=$ lipase exhaust rate $\left[\mathrm{h}^{-1}\right]$.

It has been considered the Monod forme for $\eta, \mu$ and $v_{e x}$, and Haldane forme, multiplied by $\mu$, for $\mathrm{v}_{p}$ : 


$$
\begin{aligned}
& \eta=\frac{\eta^{*} \cdot S_{1}}{K_{M_{1}}+S_{1}} \\
& \mu=\frac{\mu^{*} \cdot S_{2}}{K_{M_{2}}+S_{2}} \\
& v_{e x}=\frac{v_{e x}{ }^{*} \cdot L_{i n}}{K_{e x}+L_{i n}} \\
& v_{p}=\frac{v_{p}{ }^{*} \cdot\left(S_{1} / X\right)}{K_{p}+S_{1} / X+K_{i} \cdot\left(S_{1} / X\right)^{2}} \cdot \mu
\end{aligned}
$$

where the parameters' values are:

$$
\begin{array}{llr}
\eta^{*}=0.21 \mathrm{~h}^{-1}, & \mathrm{~K}_{\mathrm{M} 1}=0.11 \mathrm{~g} / \mathrm{l}, & \mu^{*}=0.25 \mathrm{~h}^{-1}, \\
\mathrm{~K}_{\mathrm{M} 2}=0.25 \mathrm{~g} / \mathrm{l}, & v_{\mathrm{p}}{ }^{*}=123 \mathrm{u} / \mathrm{mg}, & \mathrm{K}_{\mathrm{p}}=0.26 \mathrm{~g} / \mathrm{g}, \\
\mathrm{K}_{\mathrm{i}}=22.2 \mathrm{~g} / \mathrm{g}, & v_{\mathrm{ex}}{ }^{*}=4.09 \mathrm{~h}^{-1}, & \mathrm{~K}_{\mathrm{ex}}=19.5 \mathrm{u} / \mathrm{mg}, \\
\mathrm{Y}=1.16 \mathrm{~g} / \mathrm{g} . & &
\end{array}
$$

The model is only valid for the biomass and substrate concentration values within the range $[0-8] \mathrm{g} / \mathrm{l}$. The goal of the process control is to maximize the final lipase production.

The maximum lipase production could be obtained in two ways (Montesinos et al., 1993):

- keeping the extracellular substrate $\left(S_{1}\right)$ to a certain level (Selisteanu, 1999);

- keeping the $S_{1} / X$ ratio to a constant value, which depends on the model parameters. For the above mentioned values of the process parameters, the optimal value of the $S_{1} / X$ ratio is 0.15 .

The second variant has been adopted, where GPC algorithm controls the $S_{1} / X$ ratio. The control strategy is illustrated in Figure $18\left(N_{2}=8\right.$, $N_{u}=2, \lambda=0.5$ and $\delta=1$ ). At the beginning, the process works in open loop, with $D=0$. The biomass in the bioreactor growths on the behalf of the initial substrate. When $S_{1} / X$ becomes smaller then 0.15 , the system switches to closedloop control. The controller becomes active and controls the $S_{1} / X$ ratio, to the setpoint value 0.15 . After 22 hours, when $X$ increases over $8 \mathrm{~g} / \mathrm{l}$, the process is stopped.

In Figure 18 one can observe the two functioning regimes of the bioreactor:

- open-loop $(0<t<14 h)$;

- closed-loop $(14 h<t<22 h)$. In this case, the system works in tracking regime as the output variable $X(t)$ tracks the variable setpoint $X^{*}(t)=S_{l}(t) / 0.15$.

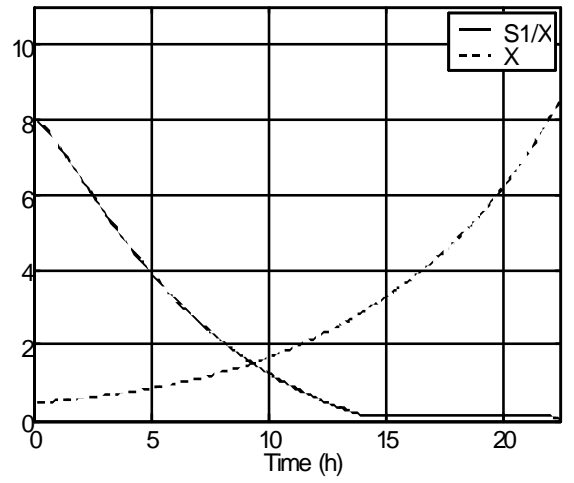

(a)

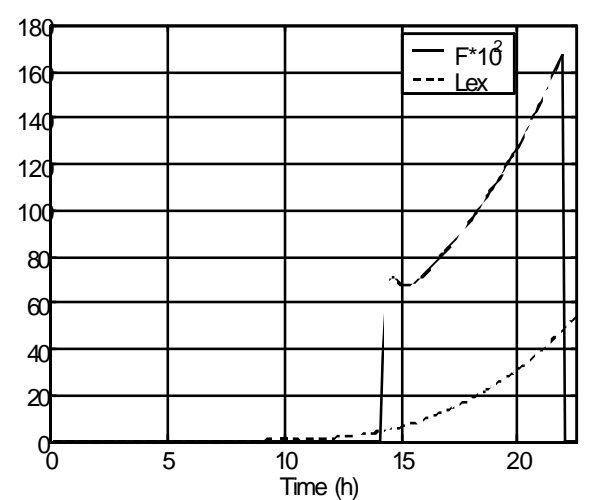

(b)

Fig. 18. a - biomass and $S_{1} / X$ ratio evolution, b the lipase and the command evolution

\section{CONCLUSIONS}

GPC structure offers good results in both cases presented in the paper: a biomass production process and a lipase production one. Comparing to IMC structure, this method offers two advantages: the neural inverting operation of the process model is eliminated and there are various possibilities to adjust the control law properties, in order to satisfy the requirements of the control feasibility.

GPC structure with discrete command leads also to good results. In this case a searching procedure for the optimal discrete command is used. This procedure could lead to a large computing time, so the control horizon should be limited $\left(N_{u} \leq 2\right)$.

\section{REFERENCES}

Aoyama, A., Venkatsubramanian, V., (1995). Inter-nal Model Control Framework Using Neural Networks for the Modeling and Control of a Bioreactor. Engng. Applic. Artif. Intell., Vol. 8, No. 6, pp. 689-701, Elsevier Science Ltd., Great Britain. 
Bastin, G. and D. Dochain (1990). On-line Estima-tion and Adaptive Control of Bioreactors, pp. 375, Elsevier.

Bhat, N. and Mcvoy, T., J. (1991). Use of Neural Nets for Dynamic Modeling and Control of Chemical Process System. AIChE J. 37, 1065-1081.

Camacho, E., F., Bordons, C., (1999). Mbdel Predictive Control, Springer-Verlag, London.

Clarke, D., W. and Mohtadi, C., (1989) Properties of Generalized Predictive Control. Automatica, 25 (vol. 6), 859-875.

De Keyser, R., (1991). Basic Principles of Model Based Predictive Control, Proc. of
European Control Conference, Grenoble, France, pp. 1753 - 1758.

Garcia, C., E. and Morari, M., (1982). Internal Model Control. A Unifying Review and Some New Results. Ind. Engng. Chem. Process Des. Dev. 25, 403-411.

Montesinos, J., L., Campmajo, C., Iza, J., Valero, F., Lafuente, J., and Sola, V., (1993). Software Development to Fermentation Gas Analysis Using Gas Spectrometer. Biotechnology techniques, Vol. 7, pp. 429-434.

Selisteanu D., (1999). Automatic Control of the Enzymatic Bioreactor, PhD Thesis, University of Craiova, Romania, pp. 65 68, $161-164$. 